\title{
CYP4F2 genetic polymorphisms are associated with coronary heart disease in a Chinese population
}

\author{
Changqing Yu ${ }^{1,2 \dagger}$, Qingkai Yan ${ }^{1,2 \dagger}$, Chunjiang Fu ${ }^{1,2}$, Weibin Shi ${ }^{1,2}$, Hongyong Wang ${ }^{1,2}$, Chunyu Zeng ${ }^{1,2}$
} and Xukai Wang ${ }^{1,2^{*}}$

\begin{abstract}
Background: To explore the relationship between CYP4F2 gene polymorphism and coronary heart disease (CHD) in a Chinese Han population.

Methods: We selected 440 CHD patients and 440 control subjects to perform a case - control study. Four SNPs (rs2108622, rs3093100, rs3093105 and rs3093135) in CYP4F2 gene were genotyped using polymerase chain reaction restriction fragment length polymorphism (PCR - RFLP) methods. The genotype and haplotype distributions were compared between the case and the control group.

Results: We found both rs2108622 and rs3093105 in CYP4F2 gene were associated with the risk for CHD $(P<0.01)$. Haplotype analysis indicated that GGGT haplotype consisted by rs2108622-rs3093100-rs3093105-rs3093135 was associated with CHD risk ( $\mathrm{OR}=4.367,95 \% \mathrm{Cl}: 2.241 \sim 8.510$; $\mathrm{P}<0.001)$, but GGTA haplotype was associated with decreased risk for $\mathrm{CHD}(\mathrm{OR}=0.450,95 \% \mathrm{Cl}: 0.111 \sim 0.777 ; P<0.001)$.
\end{abstract}

Conclusion: CYP4F2 gene polymorphisms were associated with the risk of CHD in Chinese population.

Keywords: Coronary heart disease, CYP4F2 gene, Single nucleotide polymorphism

\section{Introduction}

Coronary heart disease (CHD) is one of the most common diseases to influence the human health. Previous study indicated that the morbidity and mortality of CHD are very high [1]. CHD was a complex polygenic disease resulting from the interaction between genetic variations and environmental factors [2]. Although the exact genetic mechanism is unclear, some new susceptibility gene of the CHD have been reported recently, such as apolipoprotein (Apo) E [3], CYP8A1 [4], C5L2 [5], and interleukin (IL)-1 [6]. Grammer et al. [3] analyzed the association between the apo E genotype, CRP and angiographic coronary artery disease (CAD). The concentration of CRP was similar in patients with stable CAD and in controls, but increased

\footnotetext{
*Correspondence: wangxk_008@126.com

${ }^{\dagger}$ Equal contributors

'Department of Cardiology, Daping Hospital, The Third Military Medical University, 10\#Changjiangzhilu, Yuzhong District, Chongqing 400042, People's Republic of China

${ }^{2}$ Chongqing Institute of Cardiology, 10\#Changjiangzhilu, Yuzhong District, Chongqing 400042, People's Republic of China
}

in patients presenting with acute coronary syndromes. In models adjusting for the main confounding variables, the $\varepsilon 2$ allele was associated with a lower prevalence of angiographic CAD. Therefore, the authors concluded that the apo E genotype is associated with circulating CRP and risk for CAD. Xie et al. [4] performed a casecontrol study in a Chinese population and investigated the roles of polymorphisms in the CYP8A1 gene in CHD susceptibility. The authors found that CYP8A1 genetic polymorphisms are associated with $\mathrm{CHD}$ in Chinese population. Zheng et al. [5] identified a novel SNP-901G > A in C5L2 gene and found this SNP may be a genetic maker of CHD in the Han and Uygur population. Zhou et al. performed a meta-analysis of 13 independent case-control studies and suggested that there were no associations between IL-1 gene cluster polymorphisms and CHD. However, these genes have not been verified in different ethnicities.

20 - hydroxy eicosane arachidonic acid (20-HETE) is a metabolite of arachidonic acid (AA) produced in the 
kidney by cytochrome P450 enzymes. 20-HETE has potent actions on renal tubular and vascular function including: vasoconstriction secondary to inhibition of large conductance $\mathrm{Ca}^{2+}$-activated $\mathrm{K}^{+}$channels in vascular smooth muscle cells and inhibition of $\mathrm{Cl}^{-}$transport in the thick ascending loop of Henle [7,8]. 20-HETE has also been implicated as a second messenger mediating the inhibitory effects of dopamine, PTH, and angiotensin II on $\mathrm{Na}^{+}-\mathrm{K}^{+}$-ATPase activity and sodium transport in the proximal tubule $[9,10]$. Some studies have suggested that increased levels of 20-HETE in the renal vasculature may underlie the development of hypertension [11], and, animal experiments showed that 20-HETE can cause vasospasm after hemorrhagic stroke, and it participates in the incidence and the development process of CHD and cerebral infarction [12]. In the rat models with myocardial infarction, when the 20-HETE synthesis was suppressed, infarct size can be reduced [12]. In hypertensive mice models, the increased 20-HETE production can lead to oxidative stress and endothelial cell damage, which results in the increased incidence of CHD [13].

CYP4F2 is the major synthase which can catalyze the arachidonic acid to generate 20-HETE. Recently, Stec et al. demonstrated that a SNP (rs2108622, V433M) in CYP4F2 gene may cause 20-HETE reduction from arachidonic acid [14]. Fava et al. [15] studied the elderly patients with cerebral infarction in Sweden, and they found that V433M mutation in CYP4F2 gene was associated with cerebral infarction in male patients. A study [16] based on Japanese population found that $G$ allele frequency of rs2108622 in male patients was higher than that in the controls. TCG haplotypes composed by rs3093135rs1558139-rs2108622 is a risk factor for cerebral infarction in men [17]. In addition, Fava et al. [18] also found CYP4F2 M433 carriers had significantly higher levels of waist, triglycerides, BP and a composite sum of MetS phenotypes (MetS score) beside lower HDL-cholesterol respect to V-homozygotes. However, the relation between CYP4F2 genetic polymorphisms with CHD in Chinese population remains unclear. In the present study, we utilized a case-control study to reveal the relationship between CYP4F2 gene polymorphism and CHD.

\section{Materials and methods \\ Ethics}

The present study has been performed with the approval of the ethics committee of The Third Military Medical University and is in compliance with the Helsinki Declaration. The informed consents of the study were collected from all the candidate subjects.

\section{Subjects}

A total of 440 hospitalized CHD patients in the Department of Cardiology, Daping Hospital, The Third Military
Medical University were enrolled from September 2011 to April 2013. All these CHD patients were unrelated Han Chinese people. The patients were diagnosed CHD according to the criteria which was described previously [19]. Briefly, CHD was defined as the presence of stenosis of more than $50 \%$ luminal diameter in at least one significant coronary artery on coronary angiography. Patients with ascertained congenital hypercoagulation, with proven disease limiting life expectance or with cocaine abuse were excluded.

During the same period, 440 persons in medical center of the same hospital were selected as the control group. All these control subjects were Han people whose age and sex were matched with the patient group. The subjects with cerebrovascular disease, neurological diseases, kidney disease, blood disorders, cancer, peripheral vascular disease, and autoimmune diseases were excluded from the control group. These control subjects have no the history of CHD and any symptom of CHD. The clinical characteristics including age, gender, height, weight, blood pressure, lipids profiles, fasting glucose, past medical history, drug history, smoking history, and alcohol history were collected.

\section{DNA extraction}

$2 \mathrm{~mL}$ of fasting venous blood was taken from antecubital vein and placed in EDTA-containing tubes. The genomic DNA extraction kit (Promega Corporation, United States) was used for DNA extraction from blood samples of the subjects according to the kit's protocol.

\section{SNPs selection}

There are 740 SNPs for the human CYP4F2 gene listed in the National Center for Biotechnology Information SNP database (http://www.ncbi.nlm.nih.gov/SNP). We also screened the data for the Tag SNPs on the International HapMap Project website (http://www.hapmap.org/). We searched tag SNP using Haploview 4.2 software in human HapMap Project database with the following criteria: $\mathrm{r}^{2} \geq 0.8$ and minimum gene frequency (MAF) $\geq 0.1$. We

Table 1 Primers and PCR conditions of four SNPs

\begin{tabular}{llll}
\hline SNPs & Primers & Endonuclease & Tm $\left({ }^{\circ} \mathbf{C}\right)$ \\
\hline rs2108622 & F: 5'-ATCAACCCGTCCCACCT-3' & Pvu II & 55 \\
& R: 5'-ACATTGTGCTCCCAGACG-3' & & \\
rs3093100 & F: 5'-AGTGCTTACTAGGGAACTGGAG-3' & Apa I & 54 \\
& R: 5'-AAGGATTCAATGCAGGCCTGGA-3' & \\
rs3093105 & F: 5'-AGCCCTCCCTGCTCTACCT-3' & Eae I & 56 \\
& R:5'-CCCACTCCCTAAGCCTCGT-3'; & & \\
rs3093135 & F: 5'-GGCAGGCAGTCATCCACA-3' & Hinf I & 52 \\
& R: 5'-CCAAACAGGCCCTCACAT-3' & \\
\hline
\end{tabular}


Table 2 Characteristics of the participants population

\begin{tabular}{|c|c|c|c|c|c|c|c|c|c|c|c|c|c|}
\hline Groups & $N$ & $\begin{array}{l}\text { Age } \\
\text { (years) }\end{array}$ & $\begin{array}{l}\mathrm{BMI} \\
\mathrm{Kg} / \mathrm{m}^{2}\end{array}$ & $\begin{array}{l}\text { SBP } \\
(\mathrm{mmol} / \mathrm{L})\end{array}$ & $\begin{array}{l}\text { DBP } \\
(\mathrm{mmol} / \mathrm{L})\end{array}$ & $\begin{array}{l}\text { Hypertension } \\
(n, \%)\end{array}$ & $\begin{array}{l}\text { Diabetes } \\
(\mathrm{n}, \%)\end{array}$ & $\begin{array}{l}\text { Smoking } \\
(\mathrm{n}, \%)\end{array}$ & $\begin{array}{l}\text { GLU } \\
(\mathrm{mmol} / \mathrm{L})\end{array}$ & $\begin{array}{l}\text { TG } \\
\text { (mmol/L) }\end{array}$ & $\begin{array}{l}\mathrm{TC} \\
(\mathrm{mmol} / \mathrm{L})\end{array}$ & $\begin{array}{l}\text { HDL-C } \\
(\mathrm{mmol} / \mathrm{L})\end{array}$ & $\begin{array}{l}\text { LDL-C } \\
\text { (mmol/L) }\end{array}$ \\
\hline $\begin{array}{l}\text { HD } \\
\text { oup }\end{array}$ & 440 & $\begin{array}{l}58.6 \pm \\
13.5\end{array}$ & $\begin{array}{l}25.2 \pm \\
3.4\end{array}$ & $\begin{array}{l}143.6 \pm \\
21.1\end{array}$ & & 6) & $\begin{array}{l}139 \\
(31.6)\end{array}$ & $\begin{array}{l}105 \\
(23.9)\end{array}$ & 3.6 & $3.1 \pm 1.8$ & $5.5 \pm 2.2$ & $1.3 \pm 0.9$ & $2.6 \pm 1.4$ \\
\hline ontrol & 440 & $\begin{array}{l}58.5 \pm \\
13.2\end{array}$ & $\begin{array}{l}24.9 \pm \\
3.5\end{array}$ & $\begin{array}{l}124.2 \pm \\
13.5\end{array}$ & $\begin{array}{l}78.2 \pm \\
13.2\end{array}$ & 64 & $66(15.0)$ & 75 (17.0) & 4.6 & 1.5 & 4.0 & $1.4 \pm 0.7$ & $2.4 \pm 0.9$ \\
\hline & & 0.322 & 0.053 & $<0.001$ & $<0.001$ & $<0.001$ & 0.001 & $<0.001$ & $<0.001$ & $<0.001$ & $<0.001$ & 0.114 & 0.123 \\
\hline
\end{tabular}

Note: BMI: Body Mass Index; SBP: Systolic Blood Pressure; DBP: Diastolic Blood pressure; GLU: Glucose; TG: Triglycerides; TC: Total cholesterol; HDL-C: High-density Lipoprotein-C; LDL-C: Low-density Lipoprotein-C.

found four tag SNPs (rs3093135, rs2108622, rs3093100, and rs1558139) in CYP4F2 gene.

\section{Genotyping}

We utilized polymerase chain reaction - restriction fragment length polymorphism (PCR - RFLP) to genotype there 4 SNPs according to the protocol reported previously $[20,21]$. The primers were designed using the primer design software-primer 5.0. The primers were shown in Table 1 . To ensure the results to be verified, we used sequenced genomic DNAs as positive controls in our assays. Of the genotyped samples, $10 \%$ were duplicated and there was at least one positive and one negative control per 96-well DNA plate in our assays. The accuracy of the genotyping was determined by genotype concordance between duplicate samples. We obtained $100 \%$ concordance between the genotyped duplicate samples for each of the SNPs.

\section{Statistical analysis}

For each polymorphism, departure of the genotype distribution from that expected from Hardy-Weinberg equilibrium was assessed using the standard $\chi^{2}$ test or Fisher's exact test. Genotype frequencies in cases and controls were compared by $\chi 2$ tests. The genotype-specific risks were estimated as odds ratios (ORs). In all cases wild type genotype served as a reference group. Based on the genotype data of the genetic variations, we performed linkage disequilibrium (LD) analysis and haplotype- based case-control analysis, using the SHEsis software (http://analysis2.bio-x.cn/myAnalysis.php). In the haplotypebased case-control analysis, haplotypes with a frequency of $<0.03$ were excluded. Statistical significance was established at $\mathrm{P}<0.05$. In the present study, we performed the case-control study included $440 \mathrm{CHD}$ patients and 440 control subjects. The estimated power is $83.3 \%$.

\section{Results}

\section{The characteristics of participants}

There were no significant difference in the distribution of age, BMI, HDL-C and LDL-C between the CHD group and the control group. However, there were significant differences in hypertension, diabetes, smoking history, DBP, SBP and GLU, TG, TC concentration between CHD group and control group (Table 2).

\section{Hardy-Weinberg equilibrium}

The genotype distribution in all SNPs were in line with Hardy-Weinberg genetic equilibrium in both CHD group and the control group (all $\mathrm{P}>0.05$, data not shown).

\section{Genotype and allele frequencies}

We found GG genotype frequency of rs2108622 were significantly higher in the CHD patients than that in control group $(P<0.05)$. And the $\mathrm{G}$ allele frequency was also significantly higher than that in control group $(\mathrm{P}=0.011$, Table 3). TT genotype of rs3093105 was common in CHD patients than that in control subjects $(\mathrm{P}=0.003)$. And the

Table 3 Distributions of CYP4F2 genotypes ( $N=440)$

\begin{tabular}{|c|c|c|c|c|c|c|c|c|c|c|}
\hline \multirow[t]{2}{*}{ SNPs } & \multirow[t]{2}{*}{ Allels $(1 / 2)$} & \multirow[t]{2}{*}{ Groups } & \multicolumn{3}{|c|}{ Genotypes (n, \%) } & \multirow[t]{2}{*}{$P$ value } & \multicolumn{2}{|c|}{ Allele (n, \%) } & \multirow[t]{2}{*}{ OR $(95 \% \mathrm{Cl})$} & \multirow[t]{2}{*}{$P$ value } \\
\hline & & & $1 / 1$ & $1 / 2$ & $2 / 2$ & & 1 & 2 & & \\
\hline \multirow[t]{2}{*}{ rs2108622 } & $\mathrm{A} / \mathrm{G}$ & Case & $34(0.077)$ & $168(0.382)$ & $238(0.541)$ & 0.048 & $236(0.268)$ & $644(0.732)$ & $0.773(0.629 \sim 0.949)$ & 0.011 \\
\hline & & Control & $46(0.105)$ & $191(0.434)$ & 203(0.461) & & $283(0.322)$ & $597(0.678)$ & & \\
\hline \multirow[t]{2}{*}{ rs3093100 } & $C / G$ & Case & $7(0.016)$ & $94(0.214)$ & $339(0.770)$ & 0.482 & 108(0.123) & $772(0.877)$ & $0.877(0.664 \sim 1.158)$ & 0.357 \\
\hline & & Control & $12(0.027)$ & $97(0.220)$ & $331(0.752)$ & & $121(0.138)$ & $759(0.863)$ & & \\
\hline \multirow[t]{2}{*}{ rs3093105 } & $T / G$ & Case & $22(0.050)$ & $117(0.266)$ & $301(0.684)$ & 0.003 & $161(0.183)$ & $719(0.817)$ & $1.617(1.241 \sim 2.107)$ & $<0.001$ \\
\hline & & Control & $10(0.023)$ & 87(0.198) & $343(0.780)$ & & $107(0.122)$ & 773(0.878) & & \\
\hline \multirow[t]{2}{*}{ rs3093135 } & $\mathrm{A} / \mathrm{T}$ & Case: & $12(0.027)$ & $93(0.211)$ & $335(0.761)$ & 0.654 & $117(0.133)$ & 763(0.867) & $0.876(0.670 \sim 1.147)$ & 0.337 \\
\hline & & Control: & $15(0.034)$ & $101(0.230)$ & $324(0.736)$ & & $131(0.149)$ & $749(0.851)$ & & \\
\hline
\end{tabular}



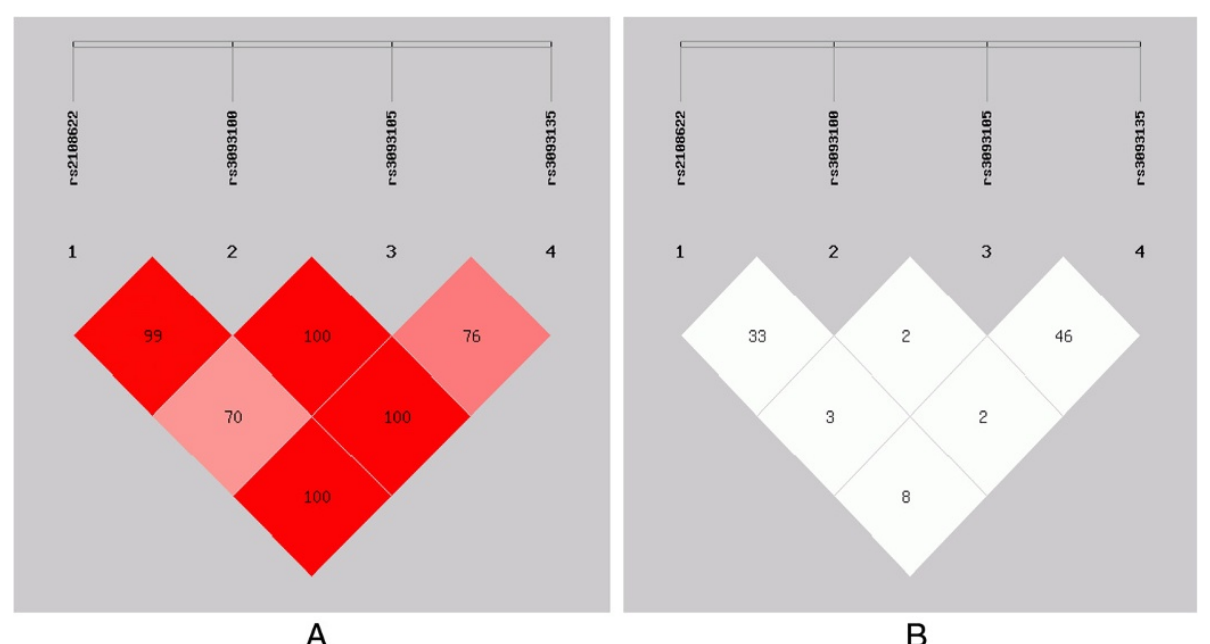

Figure 1 Genetic variation at human CYP4F2 gene. Using the SHEsis software, we calculated the Linkage disequilibrium (LD) value between each SNP. A: LD value shown: $\left|D^{\prime}\right| \times 100$; $\left|D^{\prime}\right|$ colour scheme: $\left|D^{\prime}\right|<0.5$ : white; $0.5<r^{2}<1$ : shades of Pink; $\left|D^{\prime}\right|=1$ : red; $\mathbf{B}$ : $L D$ value shown: $r^{2} \times 100$; $r^{2}$ colour scheme: $r^{2}<0.5$ : white; $0.5<r^{2}<1$ : shades of grey; $r^{2}=1$ : black.

T allele frequency was also significantly higher than that in control group $(\mathrm{P}<0.001$, Table 3$)$.

\section{Haplotype analyses}

According to the results of linkage disequilibrium analysis (As shown in Figure 1), we chose rs2108622-rs3093100rs3093105-rs3093135 to construct haplotypes using SHEsis software. The results showed that, GGGT haplotype distribution frequency in the CHD group was significantly higher than that in the control group. Individuals carrying GGGT Haplotype had 4.367 times increased risk of CHD $(\mathrm{OR}=4.367,95 \%$ CI: $2.241 \sim 8.510 ; \mathrm{P}<0.001)$. However, the GGTA frequency was lower in CHD patients than that in the control subjects. Individuals carrying GGTA Haplotype had 0.450 times decreased risk of CHD (OR = 0.450, 95\% CI: $1.111 \sim 7.777$; P < 0.001), (Table 4).

\section{Discussion}

In this haplotype-based case-control study, we found GGGT haplotype carriers have higher risk but GGTA haplotype carriers have decreased risk for CHD in Han Chinese population. To the best of our knowledge, this is the first study to reveal the relation between CYP4F2 polymorphisms and CHD in Chinese Han population.
The genetic variation is the molecular basis of human genetic diversity. The T allele of CYP4F2 rs2108622 represents a missense mutation that results in the change of valine 433 to methionine (V433M). This change in the primary structure of CYP4F2 affects enzyme activity, leading to changes in drug metabolism, physiology, and pathophysiology. Cha et al. [22] through a genome-wide association study identified rs2108622 as a genetic determinant of warfarin responsiveness for Japanese. However, no association was found between this SNP and CHD by a genome-wide association study approach. CHD is caused by the interaction between environmental and genetic factors. In this study, an important human metabolic enzyme P450 gene family member- CYP4F2 gene was selected as the candidate gene to perform the case-control study, and we found that rs2108622 and rs3093105 in CYP4F2 gene was significantly associated with higher risk for CHD. We also found GGGT haplotype consisting of rs2108622-rs3093100-rs3093105-rs3093135 was the susceptibility haplotype of CHD. Our results were consistent with the results reported by Fu et al. [17], but inconsistent with results by Ward et al. [23] and Fava et al. [15]. Ward et al. found that the A allele was a risk factor for hypertension. The other study by Fava et al. [15] found that the A allele was a risk factor for IS. This difference may be due

Table 4 Haplotype distribution between case and control

\begin{tabular}{|c|c|c|c|c|c|}
\hline Haplotypes & CHD & Control & $\mathbf{P}$ & OR & $95 \% \mathrm{Cl}$ \\
\hline$\overline{A C T T}$ & $108.00(0.123)$ & $121.00(0.137)$ & 0.310 & 0.866 & $0.656 \sim 1.144$ \\
\hline $\mathrm{A} G \mathrm{~T} T$ & $128.00(0.145)$ & $152.00(0.173)$ & 0.095 & 0.804 & $0.622 \sim 1.039$ \\
\hline$G G G A$ & $114.85(0.131)$ & $86.11(0.098)$ & 0.058 & 1.366 & $1.016 \sim 1.838$ \\
\hline G G G T & $46.14(0.052)$ & $10.89(0.012)$ & $<0.001$ & 4.367 & $2.241 \sim 8.510$ \\
\hline G GTA & $21.4(0.020)$ & $44.89(0.051)$ & $<0.001$ & 0.450 & $0.111 \sim 0.777$ \\
\hline
\end{tabular}


to different races, different methodologies and different patient selection criteria.

Singh et al. [24] found that androgen-induced CYP4A8 expression reduced $\mathrm{CYP} 2 \mathrm{C} 23$ expression and caused increased production of 20-HETE, then the epoxyeicosatrienoic acid (EET) decreased to affect the contraction of blood vessels. However, the exact mechanism of the susceptibility for CHD remains unclear.

In addition, although an elevated level of LDL-C and a decreased level of HDL-C in serum are independent risk factors for CHD, we did not find the LDL-C level and HDL-C level to be different between the CHD patients and the control subjects. This phenomenon may be the result from the treatment of decreased-cholesterol drugs (e.g., simvastatin, lovastatin) in CHD patients.

Several limitations of the present study should be noted. Firstly, these findings should be interpreted with caution because the population was only from Chinese population, this fact may reduce the possibility of confounding from ethnicity; Secondly, the present study is a hospital based case-control study, the selection bias cannot be avoidable and the subjects may not be representative of the general population; Finally, in the present study, we did not detect the amount of 20-HETE associated with CYP4F2 polymorphisms.

\section{Conclusion}

In conclusion, this study showed that both rs 2108622 and rs3093105 in CYP4F2 gene were associated with CHD in Han Chinese population.

\section{Competing interests}

The authors declared no competing interests exist.

\section{Authors' contributions}

CY and QYcarried out the molecular genetic studies and drafted the manuscript. CF and WS carried out the genotyping. HW, XW, and CZ participated in the design of the study and performed the statistical analysis. CY and CZ conceived of the study, and participated in its design and coordination and helped to draft the manuscript. All authors read and approved the final manuscript.

Received: 15 February 2014 Accepted: 14 April 2014

Published: 20 May 2014

\section{References}

1. Rossi A, Gaibazzi N, Dandale R, Agricola E, Moreo A, Berlinghieri N, Sartorio D, Loffi M, De Chiara B, Rigo F, Vassanelli C, Faggiano P: Aortic valve sclerosis as a marker of coronary artery atherosclerosis; a multicenter study of a large population with a low prevalence of coronary artery disease. Int J Cardiol 2014, 172:364-367.

2. Sayols-Baixeras S, Lluís-Ganella C, Lucas G, Elosua R: Pathogenesis of coronary artery disease: focus on genetic risk factors and identification of genetic variants. App/ Clin Genet 2014, 7:15-32.

3. Grammer TB, Hoffmann MM, Renner W, Kleber ME, Winkelmann BR, Böhm BO, März W: Apolipoprotein E genotypes, circulating C-reactive protein and angiographic coronary artery disease: the Ludwigshafen risk and cardiovascular health study. Atherosclerosis 2011, 215(2):487-493.

4. Xie X, Ma YT, Fu ZY, Yang YN, Ma X, Chen BD, Wang YH, Liu F: Association of polymorphisms of PTGS2 and CYP8A1 with myocardial infarction. Clin Chem Lab Med 2009, 47(3):347-352.

5. Zheng YY, Xie X, Ma YT, Yang YN, Fu ZY, Li XM, Ma X, Chen BD, Liu F: A novel polymorphism $(901 \mathrm{G}>\mathrm{a}$ ) of $\mathrm{C} 5 \mathrm{~L} 2$ gene is associated with coronary artery disease in Chinese Han and Uyghur population. Lipids Health Dis 2013, 12:139. doi:10.1186/1476-511X-12-139.

6. Zhou L, Cai J, Liu G, Wei Y, Tang H: Associations between interleukin-1 gene polymorphisms and coronary heart disease risk: a meta-analysis. PLoS One 2012, 7(9):e45641. doi:10.1371/journal.pone.0045641.

7. Escalante B, Erlij D, Falck JR, McGiff JC: Effect of cytochrome-P450 arachidonate metabolites on ion-transport in rabbit kidney loop of Henle. Science 1991, 251:799-802.

8. Ito O, Roman RJ: Role of 20-HETE in elevating chloride transport in the thick ascending limb of Dahl SS/Jr rats. Hypertension 1999, 33:419-423.

9. Ribeiro CM, Dubay GR, Falck JR, Mandel LJ: Parathyroid hormone inhibits Na_-K_-ATPase through a cytochrome P-450 pathway. Am J Physiol 1994, 266:F497-F505.

10. Sanchez-Mendoza A, Lopez-Sanchez P, Vazquez-Cruz B, Rios A, Martinez-Ayala $\mathrm{S}$, Escalante B: Angiotensin II modulates ion transport in rat proximal tubules through CYP metabolites. Biochem Biophys Res Commun 2000, 272:423-430.

11. Wang MH, Zhang F, Marji J, Zand BA, Nasjletti A: Laniado- Schwartzman M. CYP4A1 antisense oligonucleotide reduces mesenteric vascular reactivity and blood pressure in SHR. Am J Physiol Regul Integr Comp Physiol 2001, 280:R255-R261.

12. Liu X, Wu J, Liu H, Lai G, Zhao Y: Disturbed ratio of renal 20-HETE/EETs is involved in androgen-induced hypertension in cytochrome P450 4F2 transgenic mice. Gene 2012, 505(2):352-359. doi:10.1016/j.gene.2012.02.029.

13. Cai Y: Arachidonic acid cytochrome P450 4F2 in hypertension: what can we learn from a transgenic mouse model? Kidney Int 2009, 75(12):1253-1254. doi:10.1038/ki.2009.82.

14. Stec DE, Roman RJ, Flasch A, Rieder MJ: Functional polymorphism in human CYP4F2 decreases 20-HETE production. Physiol Genomics 2007, 30(1):74-81.

15. Fava C, Montagnana M, Almgren P, Rosberg L, Lippi G, Hedblad B, Engström G, Berglund G, Minuz P, Melander O: The V433M variant of the CYP4F2 is associated with CHD in male Swedes beyond its effect on blood pressure. Hypertension 2008, 52(2):373-380.

16. Nakamura K, Obayashi K, Araki T, Aomori T, Fujita Y, Okada Y, Kurabayashi M, Hasegawa A, Ohmori S, Nakamura T: Yamamoto K.CYP4F2 gene polymorphism as a contributor to warfarin maintenance dose in Japanese subjects. J Clin Pharm Ther 2012, 37(4):481-485.

17. Fu Z, Nakayama $T$, Sato N, Izumi $Y$, Kasamaki $Y$, Shindo A, Ohta M, Soma M, Aoi N, Sato M, Ozawa Y, Ma Y, Matsumoto K, Doba N, Hinohara S: A haplotype of the CYP4F2 gene associated with myocardial infarction in Japanese men. Mol Genet Metab 2009, 96(3):145-147.

18. Fava C, Montagnana M, Danese E, Sjögren M, Almgren P, Guidi GC, Hedblad $B$, Engström G, Minuz P, Melander O: The functional variant V433M of the CYP4F2 and the metabolic syndrome in Swedes. Prostaglandins Other Lipid Mediat 2012, 98:31-36.

19. Xie X, Ma YT, Yang YN, Fu ZY, Li XM, Huang D, Ma X, Chen BD, Liu F: Interaction between COX-2 G-765C and smoking in relation to coronary artery disease in a Chinese Uighur population. Clin Chem Lab Med 2011, 49(1):55-60.

20. Zeng WT, Zheng QS, Huang M, Cen HJ, Lai Y, Chen WY, Zhao LZ, Leng XY: Genetic polymorphisms of VKORC1, CYP2C9, CYP4F2 in Bai. Pharmazie 2012, 67(1):69-73.

21. Munshi A, Sharma V, Kaul S, Al-Hazzani A, Alshatwi AA, Shafi G, Koppula R, Mallemoggala SB, Jyothy A: Association of 1347 G/A cytochrome P450 4F2 (CYP4F2) gene variant with hypertension and stroke. Mol Biol Rep 2012, 39(2):1677-1682.

22. Cha PC, Mushiroda T, Takahashi A, Kubo M, Minami S, Kamatani N, Nakamura Y: Genome-wide association study identifies genetic determinants of warfarin responsiveness for Japanese. Hum Mol Genet 2010, 19:4735-4744.

23. Ward NC, Tsai IJ, Barden A, van Bockxmeer FM, Puddey IB, Hodgson JM, Croft KD: A single nucleotide polymorphism in the CYP4F2 but not CYP4A11 gene is associated with increased 20-HETE excretion and blood pressure. Hypertension 2008, 51(5):1393-1398.

24. Singh H, Schwartzman ML: Renal vascular cytochrome P450-derived eicosanoids in androgen-induced hypertension. Pharmacol Rep 2008, 60:29-37.

\section{doi:10.1186/1476-511X-13-83}

Cite this article as: Yu et al:: CYP4F2 genetic polymorphisms are associated with coronary heart disease in a Chinese population. Lipids in Health and Disease 2014 13:83. 